\title{
Antenatal betamethasone attenuates intrauterine infection- aggravated hyperoxia-induced lung injury in neonatal rats
}

\author{
Hye Soo Yoo', Yun Sil Chang 1,2, Jin Kyu Kim³, So Yoon Ahn', Eun Sun Kim', Dong Kyung Sung ${ }^{2}$, Ga Won Jeon', \\ Jong Hee Hwang ${ }^{5}$, Jae Won Shim ${ }^{6}$ and Won Soon Park ${ }^{1,2}$
}

BACKGROUND: Intrauterine infection can exacerbate postnatal hyperoxic lung injury. We hypothesized that antenatal betamethasone treatment attenuates hyperoxic lung injury aggravated by intrauterine infection in neonatal rats.

METHODS: Newborn Sprague-Dawley rats were divided into eight experimental groups according to (i) whether rats were exposed to normoxia (N) or hyperoxia $(\mathrm{H}, 85 \%$ oxygen) from postnatal day (P)1 to P14, (ii) whether antenatal betamethasone $(0.2 \mathrm{mg} /$ dose $)$ or vehicle was administered to pregnant rats at gestation days (E)19 and E20, and (iii) whether intrauterine infection was induced or not antenatally. Intrauterine infection was induced by intracervical inoculation of Escherichia coli into pregnant rats on E19. We measured cytokine levels of tumor necrosis factor- $\alpha$ (TNF- $\alpha$ ), interleukin (IL)-6, and IL-1 $\beta$ in P1 rat lungs and performed morphometric analyses and assessed inflammatory responses in lung tissue and bronchoalveolar lavage (BAL) at P14 by terminal deoxynucleotidyl transferase-mediated deoxyuridine triphosphate nick end labeling (TUNEL) staining and measurement of myeloperoxidase activity, collagen, and cytokine levels.

RESULTS: Cytokine levels in P1 rat lungs were increased by intrauterine infection, and these increases were attenuated by antenatal betamethasone. Hyperoxic lung injuries, indicated by morphometric changes and an inflammatory response in the lung and BAL fluid, were aggravated by intrauterine infection at P14. This aggravation was significantly attenuated by antenatal betamethasone.

CONCLUSION: Antenatal betamethasone attenuated aggravated hyperoxic lung injuries induced by intrauterine infection in neonatal rats via its anti-inflammatory actions.

B ronchopulmonary dysplasia (BPD) is one of the most important causes of mortality and long-term morbidity in very preterm infants (1). Among the multiple factors that affect the development of BPD, intrauterine infection/inflammation is known to affect the fetal lung and exacerbate postnatal evolution of BPD (2).
Corticosteroid administration to mothers in cases of imminent or anticipated preterm delivery is standard because it reduces mortality and morbidity of preterm infants (3). Chorioamnionitis is found in up to $70 \%$ of preterm deliveries before $30 \mathrm{wk}$ of gestation, and it is clinically silent in the majority of women (4). Therefore, steroid administration before delivery is relatively common clinically in cases of chorioamnionitis.

Priming of the fetal lung by chorioamnionitis is associated with increased inflammation of the fetal and/or postnatal lung (2). Inflammation is a key factor in the development of BPD (5), and steroids are potent anti-inflammatory agents (6). However, it remains unknown and controversial whether antenatal steroids prevent (7) or amplify (8) chorioamnionitisinduced complications in the fetal lung. Furthermore, it is not known whether antenatal steroids attenuate or potentiate postnatal aggravation and subsequent development of more severe BPD in fetal lungs already primed by chorioamnionitis.

Therefore, in this study, we hypothesized that antenatal betamethasone treatment would attenuate hyperoxic lung injury aggravated by intrauterine infection in neonatal rats. Neonatal rats delivered from pregnant mothers with intrauterine infections induced by cervical inoculation with Escherichia coli were subjected to prolonged postnatal exposure to hyperoxia or normoxia. We tested whether intrauterine infection would aggravate postnatal hyperoxic lung injury in newborn rats, and whether steroids given antenatally to the mothers would attenuate the aggravation of hyperoxia-induced lung injury by suppressing intrauterine inflammation or the priming of the fetal lung.

\section{RESULTS}

\section{Survival Rate and Body Weight}

Four pregnant rats per study group were used, and their mean gestational age $(\mathrm{E})$ was $22.4 \pm 0.24(\mathrm{E}=22.4 \pm 0.3, \mathrm{E}=22.5 \pm 0.4$, $\mathrm{E}=22.4 \pm 0.4$, and $\mathrm{E}=22.4 \pm 0.3$ in the control with antenatal vehicle group $(\mathrm{CV})$, control with antenatal betamethasone group (CB), intrauterine infection with antenatal vehicle group $(\mathrm{EV})$, and intrauterine infection and antenatal betamethasone

\footnotetext{
The first two authors contributed equally to this work.

'Department of Pediatrics, Samsung Medical Center, School of Medicine, Sungkyunkwan University, Seoul, South Korea; ${ }^{2}$ Samsung Biomedical Research Institute, Seoul, South Korea; ${ }^{3}$ Department of Pediatrics, School of Medicine, Chonbuk National University Hospital, Jeollabuk-do, South Korea; ${ }^{4}$ Department of Pediatrics, Pusan Paik Hospital, College of Medicine, Inje University, Pusan, South Korea; ${ }^{5}$ Department of Pediatrics, Ilsan Paik Hospital, College of Medicine, Inje University, Gyeonggi-do, South Korea; ${ }^{6}$ Department of Pediatrics, Kangbuk Samsung Hospital, School of Medicine, Sungkyunkwan University, Seoul, South Korea. Correspondence: Won Soon Park (wspark6@gmail.com)
} 
group (EB), respectively; $P>0.05)$. Litter sizes at birth ranged from 10 to 14 including stillborns $(n=12.3 \pm 1.0$ in $\mathrm{CV}, n=$ $11.8 \pm 1.7$ in $\mathrm{CB}, n=11.8 \pm 1.0$ in $\mathrm{EV}$, and $n=12.5 \pm 0.6$ in EB; $P>0.05)$. Fetal death occurred only in the E. coli-treated groups (5\%) (death/total number: 0/49,0/47, 2/47, and 3/50 in the $\mathrm{CV}, \mathrm{CB}, \mathrm{EV}$, and EB groups, respectively; $P>0.05)$.

Although the survival rate of the hyperoxic control rats with antenatal vehicle (HCV) was not significantly lower than that of the normoxic control rats with antenatal vehicle (NCV) at postnatal day (P)14, intrauterine infection significantly lowered the survival rate of the hyperoxic rats with intrauterine infection and antenatal vehicle (HEV) as compared with that of NCV rats $(P<0.05)$. Increase in the survival rate of hyperoxic rats with intrauterine infection as a result of antenatal betamethasone treatment did not reach statistical significance (hyperoxic rats with intrauterine infection and antenatal betamethasone (HEB) vs. HEV; $P>0.05$ ) (Figure 1a).

Both intrauterine infection and antenatal betamethasone treatment separately retarded intrauterine growth as compared with that of the controls $(P<0.001$ vs. $\mathrm{CV})$, with a smaller birth weight found in EV than in $\mathrm{CB}$ rats $(P<0.01)$ and, additively, with a smaller birth weight found in EB than in $\mathrm{EV}$ rats $(P<0.05)$ (Figure $\mathbf{1 b})$. After postnatal exposure to normoxia, a retarded body weight at P14 was observed in the intrauterine infection groups (normoxic rats with intrauterine infection and antenatal vehicle (NEV) and normoxic rats with intrauterine infection and antenatal betamethasone (NEB); $P<0.001$ vs. NCV) but not in the antenatal betamethasone group (normoxic control rats with antenatal betamethasone (NCB); $P>0.05$ ) as compared with that of the NCV group. Postnatal hyperoxia significantly retarded body weight gain at P14 (HCV, hyperoxic control rats with antenatal betamethasone (HCB), HEV, and HEB; $P<0.001$ vs. NCV), with a smaller body weight observed in $\operatorname{HEV}(P<0.001)$ and $\operatorname{HEB}(P<0.01)$ than in HCV rats (Figure 1c).

\section{Cytokine Levels in P1 Rat Lungs}

The concentrations of the tissue cytokines tumor necrosis factor- $\alpha$ (TNF- $\alpha$ ), interleukin (IL)-6, and IL-1 $\beta$ in the P1 rat lungs were higher in EV than in $\mathrm{CV}$ rats $(P<0.001)$. These increases were significantly attenuated by antenatal betamethasone administration (EB, $P<0.05$ vs. EV) (Figure 2).

\section{Histological Evaluation}

Fewer and larger alveoli were observed in the hyperoxia groups than in the normoxia groups, indicating hyperoxia-induced
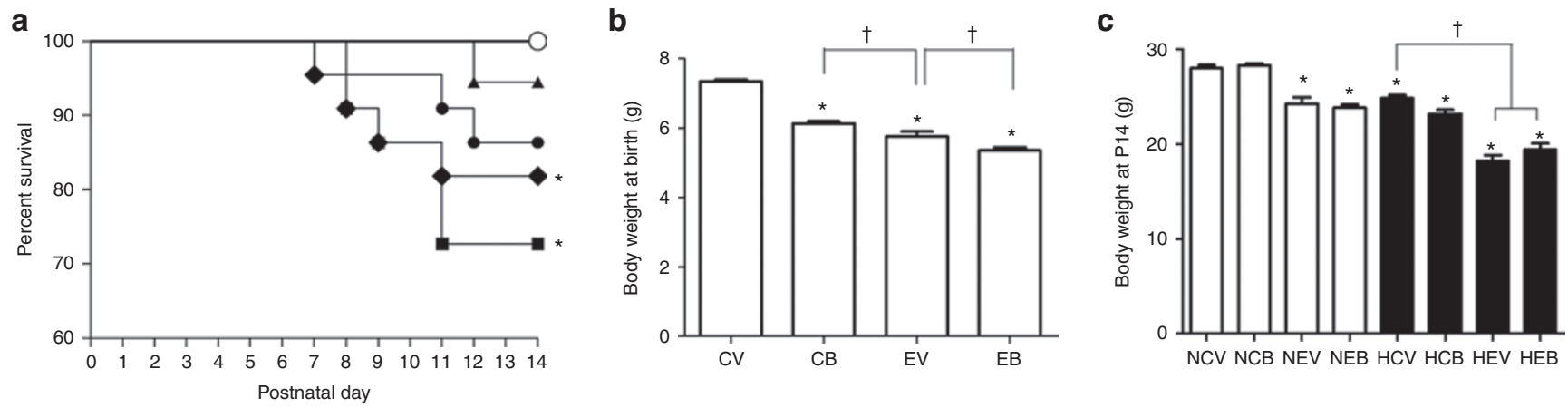

Figure 1. Survival rate and body weight. (a) Time course of survival in each experimental group in $85 \%$ oxygen or room air for $14 \mathrm{~d}$, as well as (b) birth weight and (c) body weight at postnatal day (P)14 in each experimental group. In (a), open circles: normoxia groups (NCV, NCB, NEV, and NEB); solid triangles: HCV; solid circles: HCB; solid rectangles: HEV; solid diamonds: HEB. In (b) and (c), white bars: normoxia groups; black bars: hyperoxia groups. Data are given as mean $\pm \mathrm{SEM}$. ${ }^{*} P<0.05$ vs. $\mathrm{CV}$ or NCV; ${ }^{\dagger} P<0.05$ between two groups. HCB, hyperoxic control rats with antenatal betamethasone; $\mathrm{HCV}$, hyperoxic control rats with antenatal vehicle; HEB, hyperoxic rats with intrauterine infection and antenatal betamethasone; HEV, hyperoxic rats with intrauterine infection and antenatal vehicle; NCB, normoxic control rats with antenatal betamethasone; NCV, normoxic control rats with antenatal vehicle; NEB, normoxic rats with intrauterine infection and antenatal betamethasone; NEV, normoxic rats with intrauterine infection and antenatal vehicle.

a

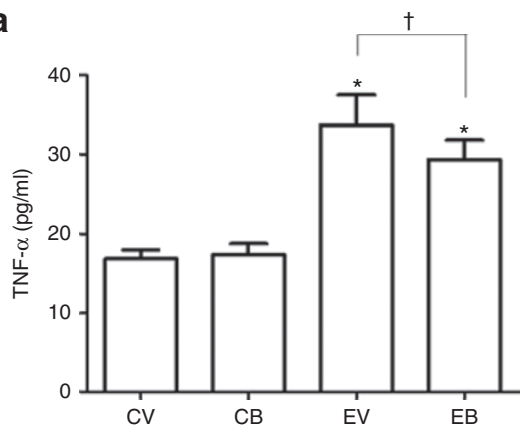

b

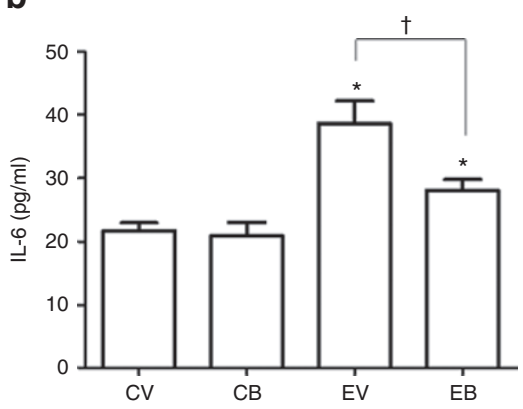

c

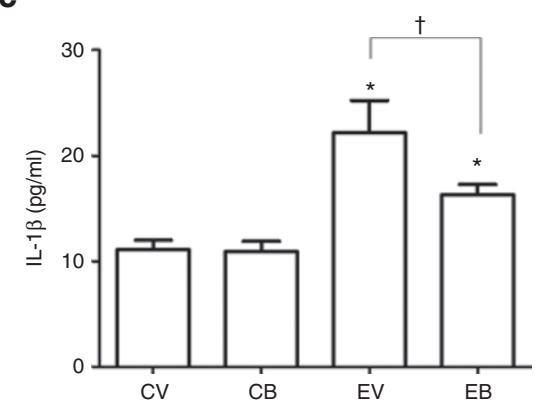

Figure 2. ELISA of (a) TNF- $\alpha$, (b) IL- 6 , and (c) IL- $1 \beta$ in $\mathrm{P} 1$ lung tissue in each experimental group. Data are given as mean \pm SEM. ${ }^{*} P<0.05$ vs. $C$; ${ }^{\dagger} P<0.05$ between two groups. $C B$, control with antenatal betamethasone group; $C V$, control with antenatal vehicle group; $E B$, intrauterine infection and antenatal betamethasone group; ELISA, enzyme-linked immunosorbent assay; EV, intrauterine infection with antenatal vehicle group; IL, interleukin; $\mathrm{P}$, postnatal day; TNF, tumor necrosis factor. 


\section{Articles | Yoo et al.}

impairment of alveolar growth. Among the hyperoxia groups, morphometric changes were aggravated in $\mathrm{HEV}$ rats, and this aggravation was significantly attenuated in $\mathrm{HEB}$ rats (Figure 3a).

In the morphometric analyses, there were no significant differences in the morphometric parameters among normoxic groups (NCV, NCB, NEV, and NEB). The mean linear intercept and the mean alveolar volume were significantly higher and the radial alveolar count was significantly lower in HCV than in NCV rats $(P<0.001)$. These hyperoxia-induced morphometric abnormalities were significantly aggravated in HEV rats $(P<0.05$ vs. HCV). Antenatal betamethasone treatment significantly attenuated the intrauterine infection-induced aggravation of disturbed alveolarization (HEB, $P<0.05$ vs. HEV for mean linear intercept and radial alveolar count; $P<0.01$ vs. $\mathrm{HEV}$ for mean alveolar volume) (Figure $3 \mathbf{b}-\mathbf{d}$ ).

Terminal Deoxynucleotidyl Transferase-Mediated Deoxyuridine Triphosphate Nick End Labeling Staining and Ectodermal Dysplasia-1-Positive Cells

There were no differences in the numbers of immunofluorescent terminal deoxynucleotidyl transferase-mediated deoxyuridine triphosphate nick end labeling (TUNEL)- and ectodermal dysplasia (ED)-1-positive cells per high-power field in the lungs of P14 rats among the normoxia groups, regardless of intrauterine infection status or antenatal betamethasone administration. The number of TUNEL- and ED-1-positive cells was greater in HCV than in NCV rats $(P<0.01$ for TUNEL; $P<0.05$ for ED-1). Among the hyperoxia groups, intrauterine infection resulted in a significant increase in the number of TUNELpositive cells (HEV, $P<0.05$ vs. $\mathrm{HCV}$ ) but not in the number of ED-1-positive cells, and antenatal betamethasone administration significantly attenuated these intrauterine infectioninduced increases in TUNEL-positive cells (HEB, $P<0.05$ vs. HEV) (Figure 4a,b).

\section{Myeloperoxidase Activity and Collagen Levels}

Myeloperoxidase (MPO) activity, as an indicator of lung neutrophil accumulation, and collagen levels, as an indicator of the degree of lung fibrosis at P14, were evaluated. There were no differences in MPO activity or collagen levels among the normoxia groups. Their levels were higher in HCV than in $\mathrm{NCV}$ rats $(P<0.001)$. Among the hyperoxia groups, both levels were significantly higher in HEV than in HCV rats $(P<$
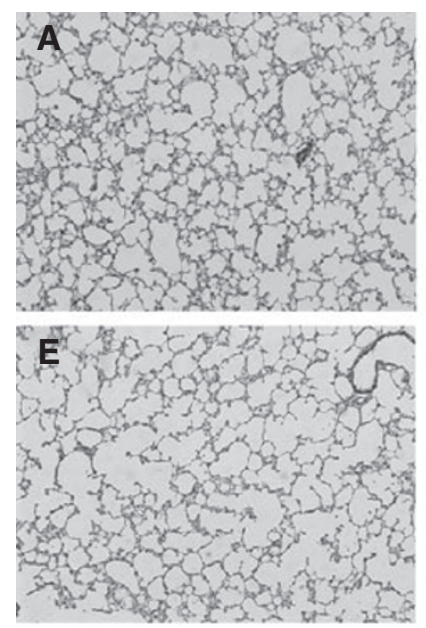
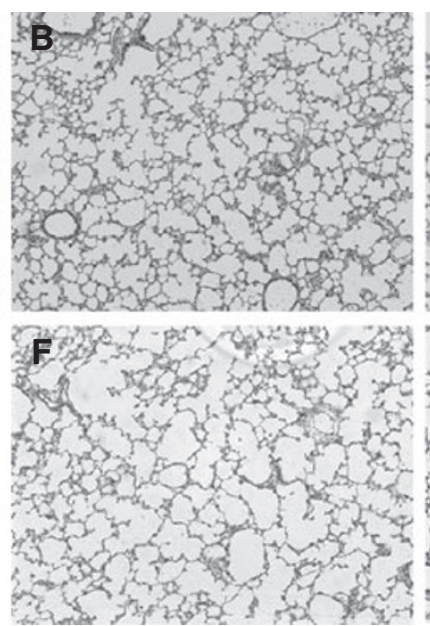
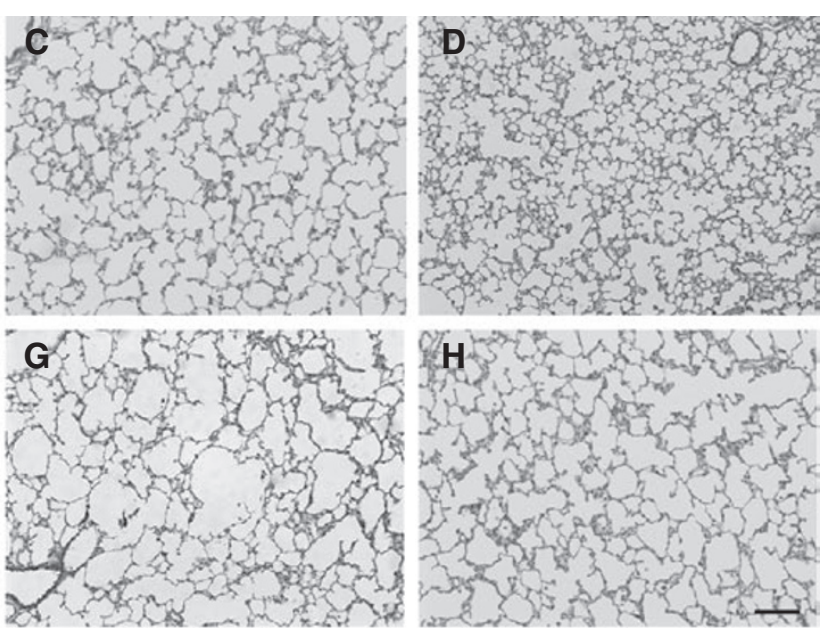

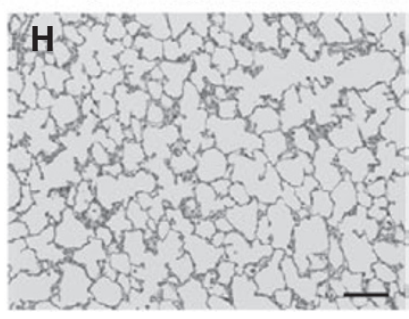

b

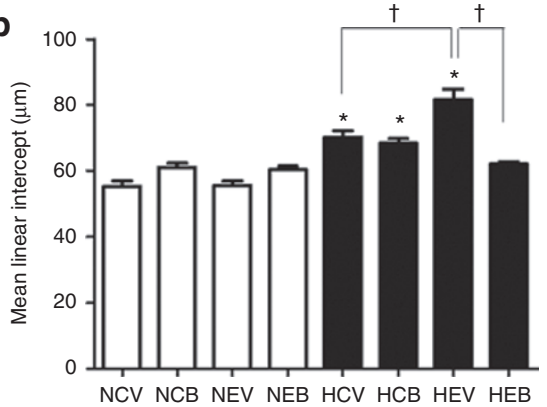

c

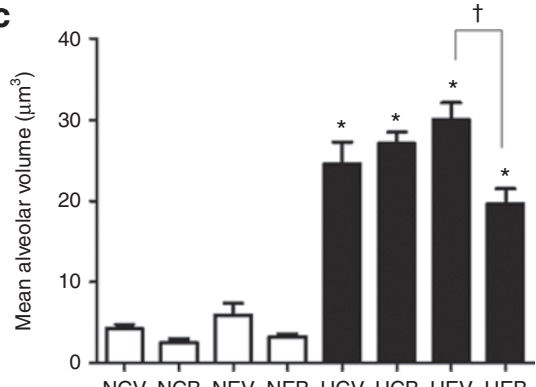

d

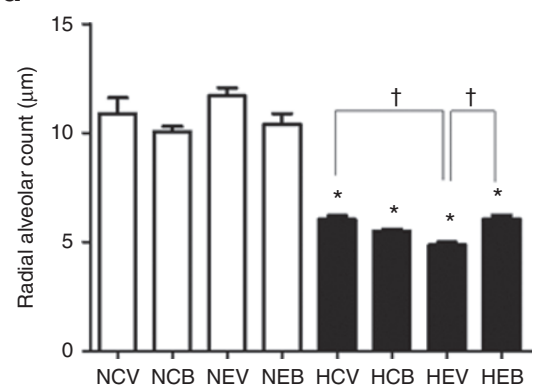

Figure 3. Histological evaluation. (a) Representative optical micrographs of rat lungs stained with hematoxylin and eosin (bar $=100 \mu \mathrm{m})$ showing the (b) mean linear intercept, (c) mean alveolar volume, and (d) radial alveolar count at P14 in each experimental group. White bars: normoxia groups; black bars: hyperoxia groups. Data are given as mean \pm SEM. ${ }^{*} P<0.05$ vs. NCV; ${ }^{+} P<0.05$ between two groups. Subpanels within (a): (A) NCV, (B) NCB, (C) NEV, (D) NEB, (E) HCV, (F) HCB, (G) HEV, (H) HEB ( $n=8,6,6,8,8,6,8$, and 8 in NCV, NCB, NEV, NEB, HCV, HCB, HEV, and HEB, respectively). HCB, hyperoxic control rats with antenatal betamethasone; $\mathrm{HCV}$, hyperoxic control rats with antenatal vehicle; $\mathrm{HEB}$, hyperoxic rats with intrauterine infection and antenatal betamethasone; $\mathrm{HEV}$, hyperoxic rats with intrauterine infection and antenatal vehicle; NCB, normoxic control rats with antenatal betamethasone; NCV, normoxic control rats with antenatal vehicle; NEB, normoxic rats with intrauterine infection and antenatal betamethasone; NEV, normoxic rats with intrauterine infection and antenatal vehicle; $P$, postnatal day. 


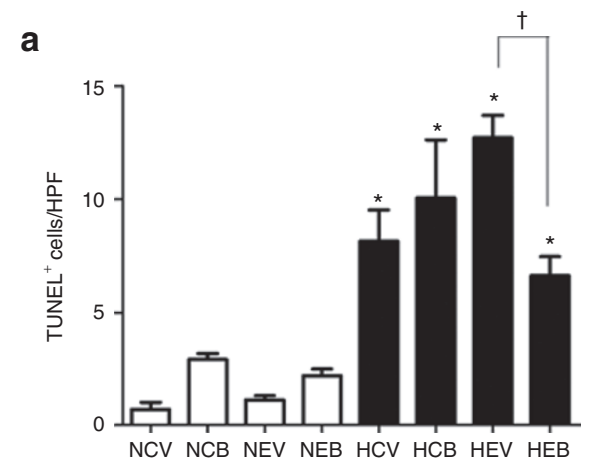

b
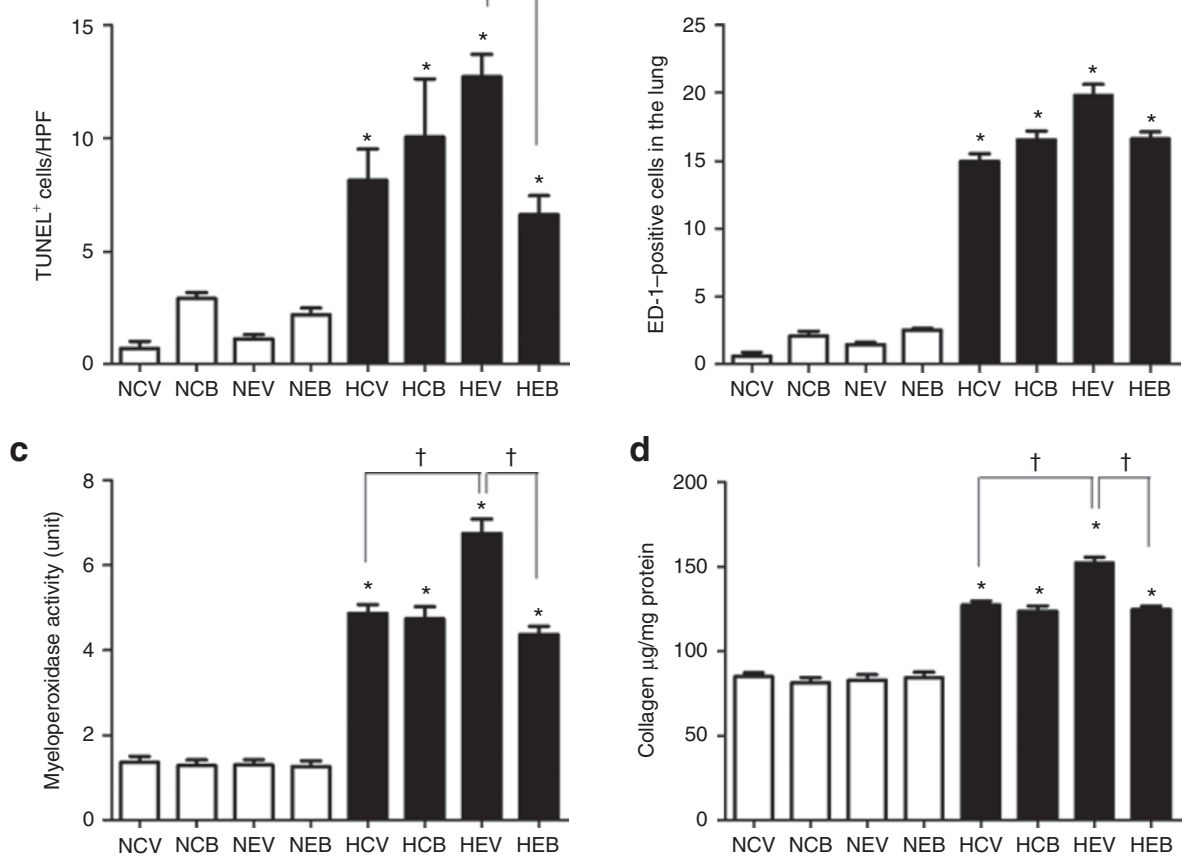

Figure 4. Number of (a) TUNEL-positive cells and (b) ED-1-positive macrophages per high-power field (HPF) $(n=6,5,6,6,7,5,8$, and 8 in NCV, NCB, NEV, NEB, HCV, HCB, HEV, and HEB, respectively), (c) myeloperoxidase activity, and (d) collagen levels in rat lung tissue at P14 in each experimental group ( $n=7,6,6,7,7,6,7$, and 7 in NCV, NCB, NEV, NEB, HCV, HCB, HEV, and HEB, respectively). White bars: normoxia groups; black bars: hyperoxia groups. Data are given as mean $\pm \mathrm{SEM}$. ${ }^{*} P<0.05 \mathrm{vs}$. $\mathrm{NCV} ;{ }^{+} P<0.05$ between two groups. ED, ectodermal dysplasia; $\mathrm{HCB}$, hyperoxic control rats with antenatal betamethasone; $\mathrm{HCV}$, hyperoxic control rats with antenatal vehicle; $\mathrm{HEB}$, hyperoxic rats with intrauterine infection and antenatal betamethasone; $\mathrm{HEV}$, hyperoxic rats with intrauterine infection and antenatal vehicle; NCB, normoxic control rats with antenatal betamethasone; NCV, normoxic control rats with antenatal vehicle; NEB, normoxic rats with intrauterine infection and antenatal betamethasone; NEV, normoxic rats with intrauterine infection and antenatal vehicle; P, postnatal day; TUNEL, terminal deoxynucleotidyl transferase-mediated deoxyuridine triphosphate nick end labeling.

0.001). Antenatal betamethasone administration significantly attenuated this intrauterine infection-induced aggravation of MPO activity and collagen levels (HEB, $P<0.001$ vs. HEV) (Figure 4c,d).

\section{White Blood Cell Counts and Cytokine Levels in Bronchoalveolar Lavage Fluid of P14 Rats}

The white blood cell counts and levels of TNF- $\alpha$, IL- 6 , and IL- $1 \beta$ in the bronchoalveolar lavage (BAL) fluid of P14 rats were measured. There were no differences among the normoxia groups. Higher white blood cell counts $(P<0.05)$ and levels of IL-6 $(P<$ $0.01)$ and IL-1 $\beta(P<0.05)$ were observed in HCV than in NCV rats. Higher level of TNF- $\alpha$ was observed only in HEV than in $\mathrm{NCV}$ rats $(P<0.001)$. Among the hyperoxia groups, the cytokine levels were significantly increased in HEV rats as compared with HCV rats $(P<0.05$ for TNF- $\alpha ; P<0.01$ for IL-6; $P<0.001$ for IL-1 $\beta$ ), and antenatal betamethasone administration significantly attenuated these intrauterine infection-induced increases (HEB, $P<0.05$ vs. HEV for TNF- $\alpha$; $P<0.001$ vs. HEV for IL-6 and IL-1 $\beta$ ) (Figure 5).

\section{DISCUSSION}

In the current study, intrauterine infection caused by intracervical E. coli inoculation not only retarded prenatal and postnatal growth but also resulted in lung inflammation at birth and subsequent aggravation of hyperoxia-induced lung injury in newborn rats. Hyperoxia-induced lung injury, as demonstrated by decreased alveolarization, a significant increase in the number of TUNEL-positive cells, and increased lung inflammation and resultant collagen deposits, was consistent with the findings in premature human infants with BPD (9), as shown in our previous studies $(10,11)$. Antenatal betamethasone administration significantly reduced lung inflammation at birth as shown by attenuated proinflammatory cytokine levels in P1 rat lungs and subsequently attenuated intrauterine infection-induced aggravation of hyperoxic neonatal lung injuries. Taken together, these findings suggest that antenatal steroids, due to their anti-inflammatory effects, can diminish the priming effects of intrauterine infections on the development of neonatal hyperoxic lung injury, i.e., BPD.

Both intrauterine infection and antenatal betamethasone administration retarded intrauterine growth separately and additively, and postnatal normalization was achieved in normoxic animals treated with antenatal betamethasone only but not in the animals with intrauterine infection, suggesting that the placental inflammatory response results in poor neonatal growth (12). In rats with intrauterine infection, hyperoxia further retarded postnatal growth and even decreased the survival rate. Antenatal betamethasone administration did not attenuate this retardation of postnatal growth and did not significantly improve the survival rate. Despite the reduction in lung inflammation and injury, the lack of a protective effect of 
a

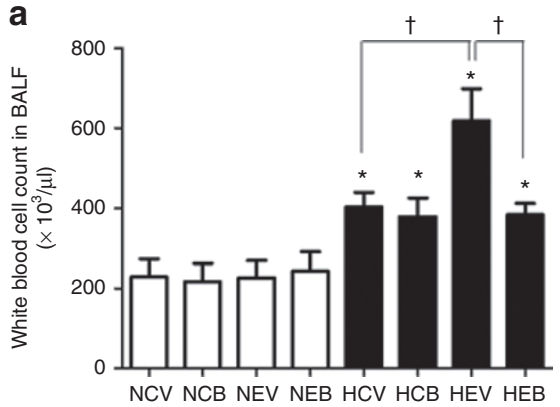

C

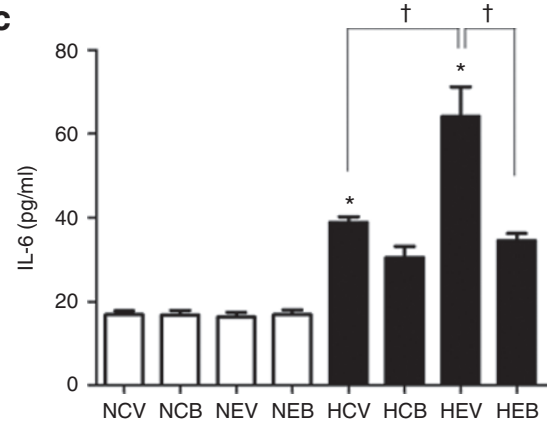

b

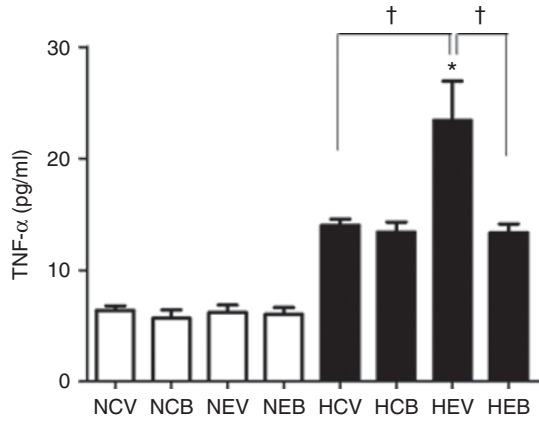

d

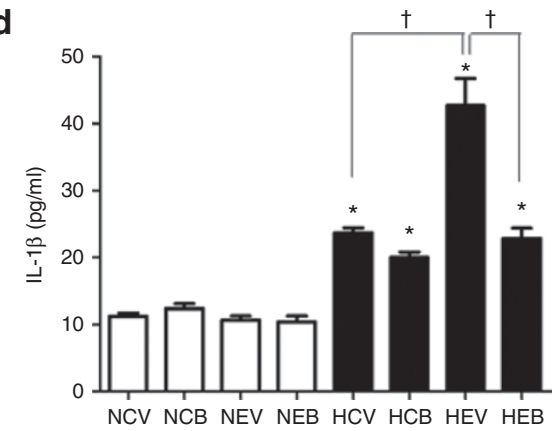

Figure 5. Inflammatory responses. (a) White blood cell count and protein levels of (b) TNF- $\alpha$, (c) IL-6, and (d) IL-1 $\beta$ in bronchoalveolar lavage fluid (BALF) at P14 in each experimental group. White bars: normoxia groups; black bars: hyperoxia groups. Data are given as mean \pm SEM. ${ }^{*} P<0.05$ vs. NCV; ${ }^{+} P<0.05$ between two groups $(n=7,6,6,7,7,6,7$, and 7 in NCV, NCB, NEV, NEB, HCV, HCB, HEV, and HEB, respectively). HCB, hyperoxic control rats with antenatal betamethasone; $\mathrm{HCV}$, hyperoxic control rats with antenatal vehicle; $\mathrm{HEB}$, hyperoxic rats with intrauterine infection and antenatal betamethasone; $\mathrm{HEV}$, hyperoxic rats with intrauterine infection and antenatal vehicle; IL, interleukin; NCB, normoxic control rats with antenatal betamethasone; $\mathrm{NCV}$, normoxic control rats with antenatal vehicle; NEB, normoxic rats with intrauterine infection and antenatal betamethasone; NEV, normoxic rats with intrauterine infection and antenatal vehicle; $P$, postnatal day; TNF, tumor necrosis factor.

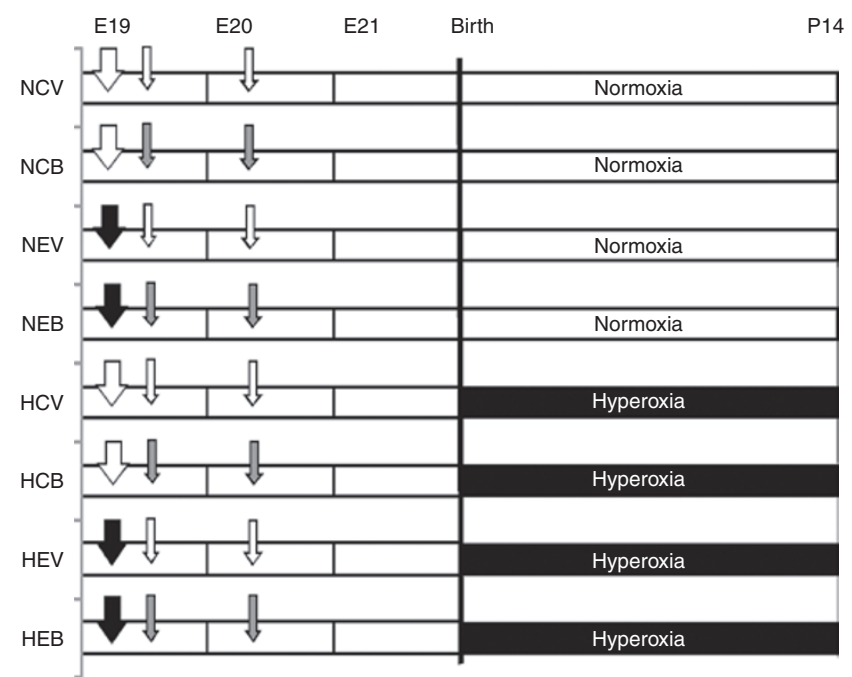

Figure 6. Schematic outline of the experimental protocol. Large white arrow: normal saline, transcervically; large black arrow: Escherichia coli, transcervically; small white arrow: normal saline, intramuscularly; small gray arrow: betamethasone, intramuscularly; normoxia: exposure to room air for $14 \mathrm{~d}$; hyperoxia: exposure to $85 \%$ oxygen for $14 \mathrm{~d}$. $\mathrm{HCB}$, hyperoxic control rats with antenatal betamethasone; $\mathrm{HCV}$, hyperoxic control rats with antenatal vehicle; $\mathrm{HEB}$, hyperoxic rats with intrauterine infection and antenatal betamethasone; $\mathrm{HEV}$, hyperoxic rats with intrauterine infection and antenatal vehicle; NCB, normoxic control rats with antenatal betamethasone; NCV, normoxic control rats with antenatal vehicle; NEB, normoxic rats with intrauterine infection and antenatal betamethasone; NEV, normoxic rats with intrauterine infection and antenatal vehicle. antenatal betamethasone on growth and survival may be a limitation of this study. However, if the rats had been exposed to lower concentrations of oxygen for more prolonged periods, or the numbers of animals in each group had been increased, the decrease in the survival rate due to hyperoxia might become significant as in other previous reports $(10,13)$, and the survival differences among the groups might be wide enough to reach statistical significance.

Live bacterial ascending infections can differ from single lipopolysaccharide (LPS)-induced(14) or IL-1-induced (15) intrauterine inflammation in that the former continuously stimulate the fetal membrane and environment. Moreover, cervical inoculation with live bacteria is similar to ascending intrauterine infection in humans, and intrauterine infection most commonly arises from the ascending route (16). Priming of the fetal lung was also demonstrated in an E. coli infection model (17) at the early gestational time point of E15 by increased proinflammatory cytokine expression in the postnatal lung, consistent with our animal model. By contrast to the report that showed a beneficial effect of moderate postnatal hyperoxia on lung growth in infant rats after exposure to intraamniotic endotoxin (18), our model of intrauterine infection at E19, which corresponds to the canalicular stage of rat lung development (19), aggravated hyperoxic lung injury due to prolonged and severe postnatal hyperoxia at P14. This finding is consistent with those of other studies, which reported that antenatal exposure of the fetal lung to endotoxins enhanced the 
subsequent inflammatory response of the postnatal lung when exposed to ventilation in preterm lambs (20) or hyperoxia in rat pups (13). Intrauterine infection and premature delivery requiring antenatal steroids in the canalicular stage of human fetal lung development (up to the 24th to 26th wk of gestation) and following exposure to hyperoxia or to a ventilator during the saccular period are relatively frequent. Therefore, our animal model is suitable for investigating lung injury in human infants born prematurely due to intrauterine infection after antenatal betamethasone treatment who are exposed to postnatal hyperoxic stimuli $(9,14)$. However, our model is limited in capacity to fully simulate BPD in human preterm infants because the rats were born at term with a short lung saccular period of 4 postnatal days (21), and exposure to severe and prolonged $85 \%$ hyperoxia is not currently commonly used in preterm infants (22).

Both chorioamnionitis and antenatal steroids improve postnatal lung function. Some studies have shown that maturational changes can occur at the expense of normal alveolar development (14); others report that antenatal betamethasone did not affect alveolarization (23) and that it even decreased both pulmonary hypertension (24) and ventilation-induced lung injury (25). Although antenatal corticosteroid administration worsened chorioamnionitis-related lung development impairment in rabbits, suggesting an additive effect on fetal lung alveolar simplification (8), betamethasone treatment after intra-amniotic LPS injection enhanced fetal lung maturation in lambs (26). In the current study, chorioamnionitis and/or antenatal steroids did not have individual or additive effects on alveolarization in P14 rat lungs exposed to normoxia postnatally. However, because we did not examine fetal or P1 rat lungs morphometrically due to technical limitations, we cannot exclude the possibility that fetal lung simplification, induced by chorioamnionitis and/or antenatal steroids, may have been reversed by normoxic exposure during postnatal development (12).

Lung inflammation initiates a progressive injury response that results in BPD (5). Antenatal steroids are potent antiinflammatory agents, and they can be used to treat the fetal lung inflammation induced by intrauterine infection. However, the interactions between antenatal steroids and chorioamnionitis are complex, and the order of exposure appears to be important. Simultaneous exposure to antenatal corticosteroids and LPS suppressed pulmonary inflammation at early stages but amplified the inflammatory response to chorioamnionitis at late stages in fetal lambs (27). By contrast, betamethasone treatment $7 \mathrm{~d}$ before intra-amniotic LPS injection suppressed lung inflammation, whereas betamethasone administration 7 $\mathrm{d}$ after LPS injection enhanced lung maturation without significant suppression of lung inflammation in fetal lambs (7). In our study, two betamethasone treatments, given at E19 and E20 after live bacterial cervical inoculation at E19, suppressed the increase in proinflammatory cytokines in P1 rat lungs. These results may be due to differences in the animal species used, the order of and interval between exposure to antenatal steroids and initiation of intrauterine inflammation, or the continuity of inflammatory stimuli by live bacteria or a single LPS injection. In the current study, the anti-inflammatory action of betamethasone might have persisted despite continuous inflammatory stimuli by live bacteria in utero before delivery, in accordance with the human study (28) showing that peak fetal serum betamethasone levels measured $1-2 \mathrm{~h}$ after maternal betamethasone treatment returned to baseline within $2 \mathrm{~d}$ of treatment.

On the other hand, it is also known that antenatal glucocorticoids upregulate the levels of the IL-6 receptor in lung epithelial cells in chorioamnionitis, leading to a subsequent induction of signal transducer and activator of transcription 3 and surfactant protein B synthesis, which can result in better lung homeostasis during an inflammatory reaction (29).

Antenatal betamethasone did attenuate the infectioninduced aggravation of hyperoxic lung injuries but did not attenuate hyperoxia-induced lung injury itself. This finding may be in accordance with the results from a recent meta-analysis showing that, in histological or clinical chorioamnionitis, antenatal steroids were not clearly associated with a reduced incidence of BPD (30). However, this might be related to the timing of the experimental interventions in our study, and further study about different timing of betamethasone treatment would be needed to clarify this.

In summary, antenatal betamethasone decreased intrauterine infection-induced fetal lung inflammation; consequently, it significantly attenuated neonatal hyperoxic lung injury aggravated by intrauterine infection in neonatal rats. Our data support the assumption that, in cases of silent or suspected chorioamnionitis, administering betamethasone to a mother with imminent or anticipated preterm delivery can suppress priming of the fetal lung and the subsequent development of more severe postnatal BPD.

\section{METHODS}

\section{Experimental Animals}

The experimental protocols described herein were reviewed and approved by the Animal Care and Use Committee of Samsung Biomedical Research Institute, Seoul, South Korea. This study also followed the institutional and US-based National Institutes of Health guidelines for laboratory animal care. As experimental animals, Sprague-Dawley newborn rat pups were randomly divided into four groups just after birth (P1) according to whether intrauterine infection was induced antenatally and whether betamethasone or vehicle was administered antenatally. The groups were named as follows: CV, $\mathrm{CB}, \mathrm{EV}$, and $\mathrm{EB}$.

Each group was then divided according to normoxia $(\mathrm{N})$ or hyperoxia $(\mathrm{H})$ into two groups for a total of eight groups: NCV, NCB, NEV, NEB, HCV, HCB, HEV, and HEB, as shown in Figure 6.

Timed-pregnant Sprague-Dawley rats were housed in individual cages with free access to water and laboratory chow, and their rat pups were delivered spontaneously. In the intrauterine infection groups, a single dose of E. coli $\left(5 \times 10^{6}\right.$ colony-forming units in $0.05 \mathrm{ml}$ of normal saline) was administered to pregnant rats intracervically on E19. Pregnant rats were anesthetized with intramuscular injection of a ketamine (45 mg/kg; Yuhan, Seoul, South Korea) and xylazine $(8 \mathrm{mg} / \mathrm{kg}$; Bayer, Leverkausen, Germany) mixture. The cervix was visualized using a speculum, and a single dose of $E$. coli was endocervically injected in the intrauterine infection groups. The same volume of normal saline without $E$. coli was injected into the control groups of pregnant rats. In our pilot study using the same methods, all amniotic fluid samples and 
$54 \%$ of cord blood samples were positive for the injected E. coli on the basis of positive cultures of these samples at the time of delivery. In the antenatal betamethasone groups, two doses of betamethasone $(0.2 \mathrm{mg}$ per dose) (betamethasone sodium phosphate; Daewon Pharmaceutical, Seoul, South Korea) were administered to pregnant rats intramuscularly at E19 and E20. In contrast to the 12-mg dose of antenatal betamethasone usually administered to pregnant women (31), a $0.2-\mathrm{mg}$ dose was administered to pregnant rats in this study. This amount was determined using a calculation based on a comparison of the body surface area of adult female rats $\left(260-280 \mathrm{~cm}^{2}\right)(32)$ with that of a human female $\left(1.73 \mathrm{~m}^{2}\right)$. Control pregnant rats that were not treated with E. coli or betamethasone received normal saline administered in the same manner. For all the groups, ceftriaxone treatment $(50 \mathrm{mg} / \mathrm{kg} / \mathrm{day}$, Triaxone injection; Hanmi Pharmaceutical, Seoul, South Korea) commenced $6 \mathrm{~h}$ after inoculation and continued every $24 \mathrm{~h}$ until delivery. The postnatal oxygen exposure (85\%) began within $10 \mathrm{~h}$ after birth and continued through P14. Throughout the experiment after deliveries, rat pups in the normoxia groups were kept with a nursing mother rat in a standard cage within a 50-1 Plexiglas chamber with room air, whereas pups in hyperoxia groups were kept with a nursing mother rat in a standard cage within the chamber in which hyperoxia (85\% oxygen concentration) was maintained. All the cages were maintained at $50 \%$ humidity and at an environmental temperature of $24^{\circ} \mathrm{C}$. To avoid oxygen toxicity, nursing mother rats were rotated daily among the litters in the normoxia and hyperoxia groups. The survival and body weight of rat pups in each group were assessed daily throughout the experiment. Rat pups were killed on $\mathrm{P} 1$ ( $n=5$ in CV, CB, EV, and EB) and $\mathrm{P} 14$ ( $n=$ 22 in NCV, $n=18$ in NCB, $n=18$ in NEV, $n=22$ in NEB, $n=22$ in $\mathrm{HCV}, n=18$ in HCB, $n=22$ in HEV, and $n=22$ in HEB) under deep pentobarbital anesthesia $(60 \mathrm{mg} / \mathrm{kg}$, intraperitoneal), and whole-lung tissue and BAL fluid were obtained.

\section{Bacterial Preparation}

We used E. coli as the source of infection because it is a common cause of intrauterine infection (33). The E. coli strain E69 was generated by $\mathrm{Pl}$ transduction of the E. coli K12 outer membrane protein $\mathrm{A}(\mathrm{Omp} A)$ gene into an OmpA- mutant of RS 218, isolated from the cerebrospinal fluid of a newborn with E. coli meningitis (a gift from Kwang Sik Kim, Johns Hopkins Hospital, Baltimore, MD) (34). E. coli was cultured overnight in $10 \mathrm{ml}$ of brain heart infusion broth (Difco Laboratories, Detroit, MI) at $37^{\circ} \mathrm{C}$. The bacteria were then diluted in brain heart infusion broth media and grown for $1 \mathrm{~h}$ to mid-logarithmic phase. The suspension was centrifuged for $10 \mathrm{~min}$ at $5,000 \mathrm{~g}$ and was washed in normal saline. The optical density was measured, and the bacteria samples were adjusted to the desired concentrations. The final E. coli preparation contained $5 \times 10^{6}$ colony-forming units in $0.05 \mathrm{ml}$ of normal saline (35).

\section{Bronchoalveolar Lavage}

Immediately after anesthesia and the exsanguination of the P14 rats, BAL was performed by placing into the trachea a 24-gauge catheter through which $2 \mathrm{ml}$ of saline was flushed back and forth three times. The BAL fluid was centrifuged, and the total and differential cell counts were obtained using a hemocytometer. The supernatants were stored at $80^{\circ} \mathrm{C}$ for cytokine analysis.

\section{Tissue Preparation}

The lungs were resected after perfusion of the heart with ice-cold phosphate-buffered saline, snap-frozen in liquid nitrogen, and stored at $-80^{\circ} \mathrm{C}$ for later biochemical analyses. For the morphometric analysis, the lungs were fixed in situ by tracheal instillation of $10 \%$ buffered formalin at a constant inflation pressure of $20 \mathrm{~cm} \mathrm{H}_{2} \mathrm{O}$ and were then fixed overnight at room temperature in the same fixative. The fixed lung tissue was embedded in paraffin wax after tissue processing. Four-micrometer-thick sections were cut from the paraffin blocks.

\section{Morphometry}

For the morphometric analysis of the lungs, the alveolarization was assessed by measuring radial alveolar count according to the method of Emery and Mithal (36); mean linear intercept was assessed as described by Cooney and Thurlbeck (37); and mean alveolar volume was calculated using the method reported by Snyder et al. (38). A minimum of two sections per rat and six fields per section were examined in each analysis, with the examiner blinded to the groups.

\section{TUNEL and ED-1 Staining}

TUNEL technique (S7110 ApopTag kit; Chemicon, Temecula, CA) was used to determine the level of apoptosis according to the manufacturer's protocol as previously described (10). The ED-1-positive cells, indicating alveolar macrophage, were identified by immunohistochemistry of the lung sections. Briefly, the tissue sections were deparaffinized and rehydrated via $20 \mathrm{~min}$ of heat-induced epitope retrieval in target retrieval solution (Dako, Glostrup, Denmark). The sections were blocked with $0.5 \%$ bovine serum albumin (Sigma-Aldrich, St. Louis, MO), and the slides were incubated overnight at $4{ }^{\circ} \mathrm{C}$ with anti-ED-1 antibody (CD68, ED-1, mouse monoclonal, hemiproteincon; Millipore, Billerica, MA). After being washed with phosphatebuffered saline, the slides were exposed for $2 \mathrm{~h}$ at room temperature to Dako polyclonal rabbit anti-mouse immunoglobulins/fluorescein isothiocyanate (1:200; Dako UK, Cambridgeshire, UK).

The slides were mounted with a vector shield mounting solution containing 4', 6-diamidino-2-phenylindole (Vector Laboratories, Burlingame, CA) and were visualized by fluorescence microscopy (Nikon E600 fluorescence microscope, Nikon, Tokyo, Japan). Ten nonoverlapping fields were examined with a magnifying power of $\times 200$ to count the TUNEL-positive and ED-1-positive cells, with the examiner blinded to the groups.

\section{MPO Activity}

The MPO activity in the homogenized lung tissue, which is an indicator of neutrophil accumulation or activity, was determined using a modification of the method reported by Gray et al. (39). One unit of MPO activity was defined as the quantity of enzyme degrading $1 \mu \mathrm{mol}$ of peroxide/min.

\section{Measurement of Collagen}

The lung collagen levels were determined from the total soluble collagen using a Sircol Collagen Assay Kit (Biocolor, Newton Abbey, UK), according to the manufacturer's instructions as previously described (11). The values in the test samples were compared with those obtained with the standard collagen solutions provided by the manufacturer, which were used to construct a standard curve.

\section{Enzyme-Linked Immunosorbent Assay}

The frozen lungs at P1 were homogenized in cold buffer $(50 \mathrm{mmol} / \mathrm{l}$ Tris-HCl, pH 7.4) with $1 \mathrm{mmol} / \mathrm{l}$ EDTA, $1 \mathrm{ml}$ of ethyleneglycolbis(baminoethylether)-N,N9-tetraacetic acid, $1 \mathrm{ml}$ phenylmethanesulfonyl fluoride, $42 \mathrm{mmol} / \mathrm{l} \mathrm{KCl}$, and $5 \mathrm{mmol} / \mathrm{l} \mathrm{MgCl}_{2}$. The samples were centrifuged at $8,000 \mathrm{~g}$ for $20 \mathrm{~min}$ at $4{ }^{\circ} \mathrm{C}$ to remove any cellular debris. BAL fluid obtained from P14 rats was centrifuged at $450 \mathrm{~g}$ for $10 \mathrm{~min}$. The protein content in the supernatant of homogenized lung BAL fluid was measured using the Bradford method with a bovine serum albumin standard. TNF- $\alpha$, IL- 6 , and IL- $1 \beta$ were measured using the Milliplex MAP ELISA Kit (Millipore) according to the manufacturer's instructions.

\section{Statistical Analyses}

The data are expressed as mean \pm SEM. Survival rates were compared using Kaplan-Meier analysis followed by a log-rank test. Comparisons among groups were made using mixed model with "group" as fixed and "mother rat" as random effect for the consideration of pregnancies from which subjects originated, followed by post hoc multiple comparisons by the Tukey method. All data were analyzed using the SAS Enterprise Guide 3.0 (SAS Institute, Cary, NC). $P$ values $<0.05$ were considered significant.

\section{STATEMENT OF FINANCIAL SUPPORT}

This work was supported by the Basic Science Research Program through the National Research Foundation of Korea and was funded by the Ministry of Education, Science, and Technology (grant S-2011-0014046) and by a Samsung Biomedical Research Institute grant (SBRI CB11271). 


\section{REFERENCES}

1. Bhandari A, Panitch HB. Pulmonary outcomes in bronchopulmonary dysplasia. Semin Perinatol 2006;30:219-26.

2. Kramer BW. Antenatal inflammation and lung injury: prenatal origin of neonatal disease. J Perinatol 2008;28:Suppl 1:S21-7.

3. Roberts D, Dalziel S. Antenatal corticosteroids for accelerating fetal lung maturation for women at risk of preterm birth. Cochrane Database Syst Rev 2006:CD004454

4. Goldenberg RL, Culhane JF, Iams JD, Romero R. Epidemiology and causes of preterm birth. Lancet 2008;371:75-84.

5. Kallapur SG, Jobe AH. Contribution of inflammation to lung injury and development. Arch Dis Child Fetal Neonatal Ed 2006;91:F132-5.

6. Doyle LW, Ehrenkranz RA, Halliday HL. Dexamethasone treatment after the first week of life for bronchopulmonary dysplasia in preterm infants: a systematic review. Neonatology 2010;98:289-96.

7. Kuypers E, Collins JJ, Kramer BW, et al. Intra-amniotic LPS and antenatal betamethasone: inflammation and maturation in preterm lamb lungs. Am J Physiol Lung Cell Mol Physiol 2012;302:L380-9.

8. Joram N, Launay E, Roze JC, et al. Betamethasone worsens chorioamnionitis-related lung development impairment in rabbits. Am J Perinatol 2011;28:605-12.

9. Coalson JJ. Pathology of new bronchopulmonary dysplasia. Semin Neonatol 2003;8:73-81.

10. Chang YS, Choi SJ, Sung DK, et al. Intratracheal transplantation of human umbilical cord blood-derived mesenchymal stem cells dose-dependently attenuates hyperoxia-induced lung injury in neonatal rats. Cell Transplant 2011;20:1843-54.

11. Chang YS, Oh W, Choi SJ, et al. Human umbilical cord blood-derived mesenchymal stem cells attenuate hyperoxia-induced lung injury in neonatal rats. Cell Transplant 2009;18:869-86.

12. Jobe AH. Antenatal factors and the development of bronchopulmonary dysplasia. Semin Neonatol 2003;8:9-17.

13. Kim DH, Choi CW, Kim EK, et al. Association of increased pulmonary interleukin-6 with the priming effect of intra-amniotic lipopolysaccharide on hyperoxic lung injury in a rat model of bronchopulmonary dysplasia. Neonatology 2010;98:23-32.

14. Willet KE, Jobe AH, Ikegami M, Newnham J, Brennan S, Sly PD. Antenatal endotoxin and glucocorticoid effects on lung morphometry in preterm lambs. Pediatr Res 2000;48:782-8.

15. Willet KE, Kramer BW, Kallapur SG, et al. Intra-amniotic injection of IL-1 induces inflammation and maturation in fetal sheep lung. Am J Physiol Lung Cell Mol Physiol 2002;282:L411-20.

16. Grigsby PL, Novy MJ, Adams Waldorf KM, Sadowsky DW, Gravett MG. Choriodecidual inflammation: a harbinger of the preterm labor syndrome. Reprod Sci 2010;17:85-94.

17. Zhan CY, Yuan TM, Sun Y, Yu HM. Early gestational intrauterine infection induces postnatal lung inflammation and arrests lung development in a rat model. J Matern Fetal Neonatal Med 2011;24:213-22.

18. Tang JR, Seedorf GJ, Muehlethaler V, et al. Moderate postnatal hyperoxia accelerates lung growth and attenuates pulmonary hypertension in infant rats after exposure to intra-amniotic endotoxin. Am J Physiol Lung Cell Mol Physiol 2010;299:L735-48.

19. Gomi T, Kimura A, Adriaensen D, et al. Stages in the development of the rat lung: morphometric, light and electron microscopic studies. Kaibogaku Zasshi 1994;69:392-405.
20. Ikegami M, Kallapur SG, Jobe AH. Initial responses to ventilation of premature lambs exposed to intra-amniotic endotoxin 4 days before delivery. Am J Physiol Lung Cell Mol Physiol 2004;286:L573-9.

21. Burri PH. Structural aspects of postnatal lung development - alveolar formation and growth. Biol Neonate 2006;89:313-22.

22. Jobe AH. The new bronchopulmonary dysplasia. Curr Opin Pediatr 2011;23:167-72.

23. San Feliciano L, Remesal A, Isidoro-García M, Ludeña D. Dexamethasone and betamethasone for prenatal lung maturation: differences in vascular endothelial growth factor expression and alveolarization in rats. Neonatology 2011;100:105-10.

24. Roubliova XI, Van der Biest AM, Vaast P, et al. Effect of maternal administration of betamethasone on peripheral arterial development in fetal rabbit lungs. Neonatology 2008;93:64-72.

25. Hillman NH, Pillow JJ, Ball MK, Polglase GR, Kallapur SG, Jobe AH. Antenatal and postnatal corticosteroid and resuscitation induced lung injury in preterm sheep. Respir Res 2009;10:124.

26. Sweet DG, Huggett MT, Warner JA, et al. Maternal betamethasone and chorioamnionitis induce different collagenases during lung maturation in fetal sheep. Neonatology 2008;94:79-86.

27. Kallapur SG, Kramer BW, Moss TJ, et al. Maternal glucocorticoids increase endotoxin-induced lung inflammation in preterm lambs. Am J Physiol Lung Cell Mol Physiol 2003;284:L633-42.

28. Ballard PL, Granberg P, Ballard RA. Glucocorticoid levels in maternal and cord serum after prenatal betamethasone therapy to prevent respiratory distress syndrome. J Clin Invest 1975;56:1548-54.

29. Ladenburger A, Seehase M, Kramer BW, et al. Glucocorticoids potentiate IL-6-induced SP-B expression in H441 cells by enhancing the JAKSTAT signaling pathway. Am J Physiol Lung Cell Mol Physiol 2010;299: L578-84.

30. Been JV, Degraeuwe PL, Kramer BW, Zimmermann LJ. Antenatal steroids and neonatal outcome after chorioamnionitis: a meta-analysis. BJOG 2011;118:113-22.

31. Peltoniemi OM, Kari MA, Hallman M. Repeated antenatal corticosteroid treatment: a systematic review and meta-analysis. Acta Obstet Gynecol Scand 2011;90:719-27.

32. Samuel L. The determination of the surface area of the white rat. J Nutr 1930;3:7.

33. Gibbs RS, Duff P. Progress in pathogenesis and management of clinical intraamniotic infection. Am J Obstet Gynecol 1991;164(5 Pt 1):1317-26.

34. Salminen A, Paananen R, Vuolteenaho R, et al. Maternal endotoxininduced preterm birth in mice: fetal responses in toll-like receptors, collectins, and cytokines. Pediatr Res 2008;63:280-6.

35. Kramer BW, Moss TJ, Willet KE, et al. Dose and time response after intraamniotic endotoxin in preterm lambs. Am J Respir Crit Care Med 2001;164:982-8.

36. Cooney TP, Thurlbeck WM. The radial alveolar count method of Emery and Mithal: a reappraisal 1-postnatal lung growth. Thorax 1982;37:572-9.

37. Cooney TP, Thurlbeck WM. The radial alveolar count method of Emery and Mithal: a reappraisal 2-intrauterine and early postnatal lung growth. Thorax 1982;37:580-3.

38. Snyder JM, Jenkins-Moore M, Jackson SK, et al. Alveolarization in retinoic acid receptor-beta-deficient mice. Pediatr Res 2005;57:384-91.

39. Gray KD, Simovic MO, Chapman WC, et al. Endotoxin potentiates lung injury in cerulein-induced pancreatitis. Am J Surg 2003;186:526-30. 